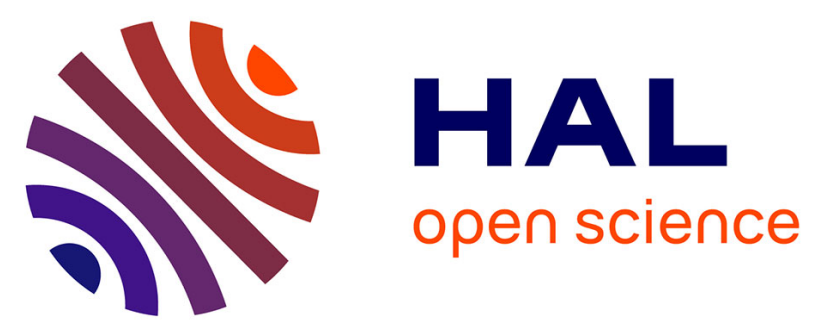

\title{
ÉTUDE DES DÉFAUTS D'EMPILEMENT DANS LES ALLIAGES CUBIQUES A FACES CENTRÉES DU NICKEL
}

Pierre Coulomb, Nicole Gibert, Alain Clément, Armand Coujou

\section{To cite this version:}

Pierre Coulomb, Nicole Gibert, Alain Clément, Armand Coujou. ÉTUDE DES DÉFAUTS D'EMPILEMENT DANS LES ALLIAGES CUBIQUES A FACES CENTRÉES DU NICKEL. Journal de Physique Colloques, 1966, 27 (C3), pp.C3-94-C3-97. 10.1051/jphyscol:1966311 . jpa-00213121

HAL Id: jpa-00213121

https://hal.science/jpa-00213121

Submitted on 1 Jan 1966

HAL is a multi-disciplinary open access archive for the deposit and dissemination of scientific research documents, whether they are published or not. The documents may come from teaching and research institutions in France or abroad, or from public or private research centers.
L'archive ouverte pluridisciplinaire HAL, est destinée au dépôt et à la diffusion de documents scientifiques de niveau recherche, publiés ou non, émanant des établissements d'enseignement et de recherche français ou étrangers, des laboratoires publics ou privés. 


\title{
ÉTUDE DES DÉFAUTS D'EMPILEMENT DANS LES ALLIAGES CUBIQUES A FACES CENTRÉES DU NICKEL
}

\author{
par Pierre Coulomb, Nicole Gibert, Alain Clément et Armand Coujou. \\ Laboratoire de Physique Electronique - Etude du Métal, \\ Faculté des Sciences de Toulouse.
}

\begin{abstract}
Résumé. - Une étude radiocristallographique (Méthode de Paterson à $-150^{\circ} \mathrm{C}$ ) et la détermination de la fréquence des macles de recuit ont permis de sélectionner quelques alliages $\mathrm{c}$. f. $\mathrm{c}$. du nickel dont l'énergie de défaut pouvait être nettement inférieure à celle du nickel pur. D'après le rayon de courbure des partielles des nouds élargis mesuré au microscope électronique, on trouve une énergie de défaut intrinsèque de $80 \mathrm{erg} / \mathrm{cm}^{2}$ environ pour $\mathrm{Ni}-39 \% \mathrm{Cr}$ ou $\mathrm{Ni}-17 \% \mathrm{Mo}-5 \% \mathrm{Fe}$, alors que cette énergie n'est que de $20 \mathrm{erg} / \mathrm{cm}^{2}$ pour un acier inoxydable. Cette étude souligne l'importance du choix et du nombre des nœuds (isolés ou en réseau), de la formule et des constantes élastiques utilisées, et surtout du traitement mécanique et thermique qui a formé les nœuds dissociés.

Abstract. - X-ray studies (Paterson's method at $-150^{\circ} \mathrm{C}$ ) and determinations of the frequency of annealing twins have permitted the selection of a few f. c. c. nickel base alloys, the stacking fault energy of which might be considerably lower than that of. Curvature radii of extended node partials measured in an electron microscope, give as intrinsic fault energy about $80 \mathrm{erg} / \mathrm{cm}^{2}$ for $\mathrm{Ni}-39 \% \mathrm{Cr}$ or $\mathrm{Ni}-17 \% \mathrm{Mo}-5 \% \mathrm{Fe}$, whilst this energy is only $20 \mathrm{erg} / \mathrm{cm}^{2}$ for a stainless steel. The study points out the importance of selection and number of nodes (isolated or in a network), of formula and elastic constants, and especially of thermal and mechanical treatment which has produced the extended nodes.
\end{abstract}

Nous avons entrepris l'étude de l'influence de divers éléments sur la présence et l'énergie des défauts d'empilement dans le nickel. La donnée la plus intéressante à connaître est l'énergie spécifique de défaut d'empilement intrinsèque $\gamma$. Comme la dissociation d'une dislocation en deux imparfaites séparées par une bande de défaut est inobservable dans ces alliages, la méthode la plus sûre pour estimer cette énergie est l'étude au microscope électronique de nouds de dislocations élargis; elle ne s'applique cependant qu'aux alliages dont l'énergie de défaut, ou plutôt dont le rapport de l'énergie de défaut au module de cisaillement est relativement faible, ce qui n'est pas le cas pour le nickel pur.

Plutôt que de mettre au point pour chaque alliage une méthode d'amincissement électrolytique et de rechercher au microscope électronique des nouds après divers traitements, nous avons fait appel à la méthode radiocristallographique de Paterson pour sélectionner les alliages qui, présentant d'assez nombreux défauts, doivent avoir une énergie $\gamma$ relativement faible. Les défauts d'empilement déplacent dans des sens opposés les raies de diffraction $\mathrm{X}$, et l'on peut estimer d'après la variation de distance angulaire des pics la proportion $\alpha$ de la surface en défaut d'empilement par rapport à la surface totale des plans $\{111\}$. Pour que la méthode soit sensible, il faut déformer considérablement le métal pour introduire beaucoup de défauts, et le maintenir à une température assez basse pour qu'ils ne disparaissent pas : nous avons limé les alliages sous azote liquide et maintenu la limaille à $-150^{\circ} \mathrm{C}$ pendant la diffraction. Des mesures point par point avec un compteur à scintillation nous ont permis d'évaluer le déplacement des pics de diffraction $\{111\}$ et $\{200\}$ quand on passe du métal limé sous azote au métal recuit [1]. La probabilité de défaut est dans ces conditions de 0,003 pour le nickel pur, elle s'élève à 0,008 pour $0,5 \%$ de titane, $2 \%$ de silicium, $7 \%$ de molybdène ou $18 \%$ de chrome; elle atteint 0,015 pour $4 \%$ de silicium, $39 \%$ de chrome ou $17 \%$ de molybdène $+5 \%$ de fer (tous les pourcentages de cette communication sont indiqués en masse). Ces probabilités s'élèvent én général a vec la teneur en élément d'alliage, mais les valeurs atteintes sont très différentes suivant les propriétés de celui-ci, en particulier sa valence [2] 


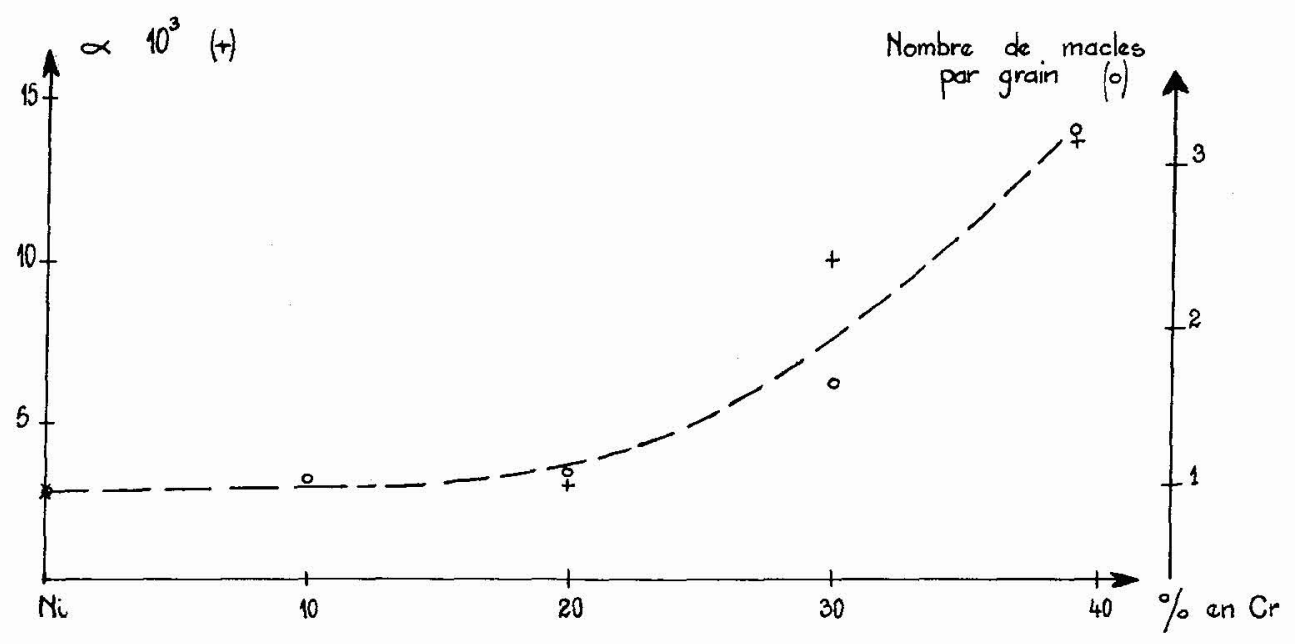

FIG: 1. - Probabilité de faute $\alpha$ à $-150^{\circ}$

et nombre de macles de recuit par grain dans les alliages nickel-chrome.

et sa solubilité dans le nickel. $\alpha$ peut rester très faible même pour des éléments miscibles avec le nickel en toute proportion. Ainsi un alliage à $50 \%$ de palladium ne présente qu'une probabilité de défaut de 0,006 après limage sous azote liquide, un alliage à $50 \%$ de cuivre ou un alliage à $15 \%$ de rhodium donne dans ces conditions le même chiffre que le nickel pur $(0,003)[3]$.

Pour rechercher rapidement les éléments qui modifient nettement l'énergie de défaut, on peut aussi mesurer la fréquence des macles de recuit [4]-[5] dans des conditions d'opération bien définies et telles que cette fréquence ne varie plus sensiblement avec le temps; nous effectuons ainsi un laminage de $60 \%$, puis un recuit de $80 \mathrm{mn}$ à $1030^{\circ}$. La figure 1 montre par exemple que le nombre de macles par grain, déterminé par micrographie optique, s'élève de 1,1 à 3,2 quand la teneur en chrome passe de 0 à $39 \%$, mais que l'effet de cet élément ne se fait vraiment sentir qu'au-dessus de $20 \%$, comme l'indiquait la diffraction des rayons $X$.

Ces deux méthodes ont l'avantage de classer rapidement des alliages, même si leur énergie de défaut est élevée, et s'ils ne donnent aucune dissociation de nœuds de dislocations perceptible au microscope électronique. Seule l'étude des nœuds nous a cependant permis de donner une estimation de l'énergie spécifique des défauts $\gamma$. Des méthodes d'approximations successives ou de calcul variationnel [6 à 9] tirent en effet $\gamma / G$ de la forme des dislocations imparfaites qui bordent un nœud symétrique dans un métal supposé isotrope. Nous avons mesuré le rayon de courbure des imparfaites sur des photographies (grandissement sur plaque environ 20000 , sur papier environ 250000 ). La mesure du rayon du cercle inscrit dans le noud devient en effet tout à fait inexacte lorsque le nœud se rétrécit et que le décalage de l'image d'une imparfaite due à la diffraction électronique est de l'ordre de grandeur du rayon mesuré.

La mise en œuvre expérimentale pose cependant de nombreux problèmes : un aspect essentiel est le traitement thermique et mécanique qui a produit les nœuds, puis les a parfois modifiés. Dans un alliage $\mathrm{Ni}-39 \% \mathrm{Cr}$ trempé depuis $1000^{\circ} \mathrm{C}$ on observe des nouds de $140 \AA$ de rayon de courbure (21 mesures). Une traction de $1,5 \%$ effectuée sur le même métal recuit donne des rayons de courbure de $197 \AA$ (96 mesures) (Fig. 2); avec un coefficient de Pois-

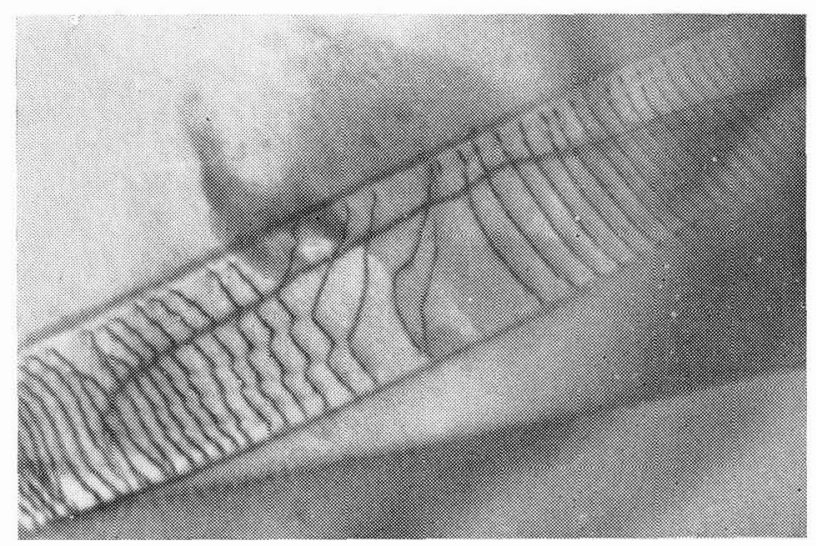

FIG. 2. - Alliage nickel-39\% chrome (après traction de 1,5\%) (X 80000$)$. 


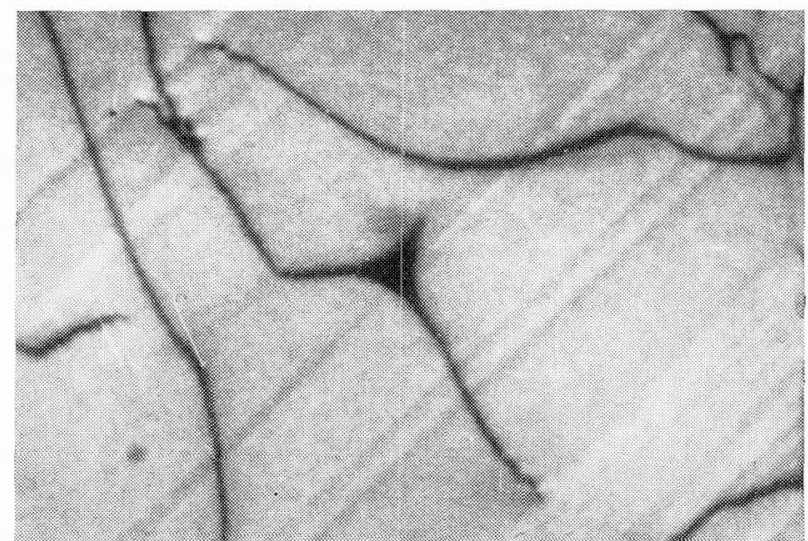

a) isolé (X 80000$)$

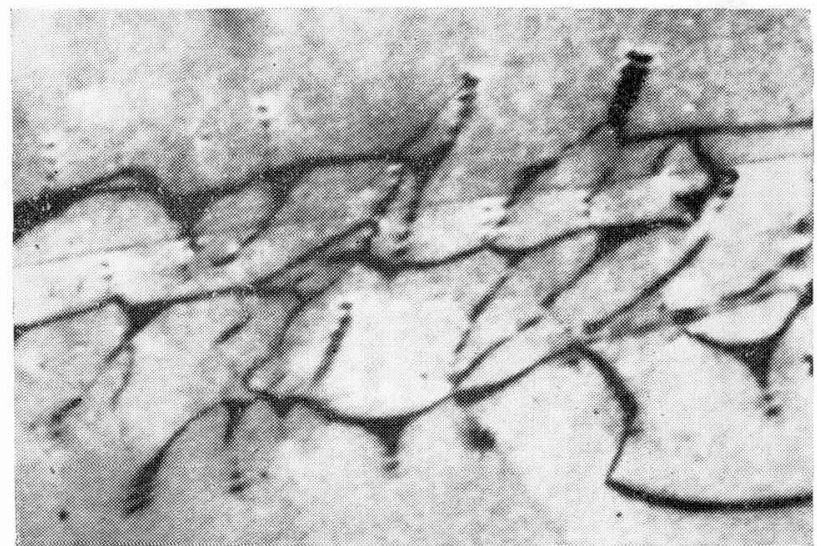

b) dans un réseau (X 80000$)$

FIG. 3. - Nouds dans l'acier inoxydable

son de 0,31 et un module de cisaillement de $8,2 \times 10^{11}$ dyne $/ \mathrm{cm}^{2}$ [10], la formule de Brown et Thölen donne des énergies $\gamma$ de 107 et $81 \mathrm{erg} / \mathrm{cm}^{2}$ respectivement. Le chiffre obtenu après une faible déformation à la température ambiante ne peut être modifié que par quelques contraintes résiduelles et doit être préféré. Le nœud produit à température élevée correspond à un équilibre des imparfaites en présence d'un effet Suzuki de ségrégation et avec un module de cisaillement $G$ très réduit (celui $\mathrm{du}$ nickel vaut ainsi $7,1 \times 10^{11}$ dyne $/ \mathrm{cm}^{2}$ à l'ambiante et $3,7 \times 10^{11}$ à $1000^{\circ} \mathrm{C}$ ); la ségrégation a pu fixer, au moins en partie, la forme du nœud de haute température si bien que dans le cas particulier envisagé, les chiffres obtenus ne nous permettent pas d'affirmer si $\gamma$ croît ou décroît quand la température s'élève.

Le choix parmi les nœuds observés sur un alliage est lui aussi important. Dans un acier inoxydable $(16,6 \% \mathrm{Cr}, 9,5 \% \mathrm{Ni}, 0,028 \% \mathrm{C})$ les nœuds sont nombreux et nous avons mesuré séparément les rayons de courbure des nœuds isolés (48 mesures) et des nœuds faisant partie d'un réseau plus ou moins irrégulier de dislocations (68 mesures). La différence des rayons (Fig. $3 a$ et $3 b$ ) est très significative $880 \AA$ dans le premier cas, $585 \AA$ dans le second, avec les constantes élastiques de cet acier $\gamma=19$ et $27 \mathrm{erg} / \mathrm{cm}^{2}$ respectivement. Nous devons prendre le premier chiffre qui exclut l'interaction avec des dislocations assez proches, mais quand les nœuds sont rares dans un alliage on est amené à considérer aussi les nœuds de réseau.

L'application de la formule de Brown et Thölen nécessite, outre la connaissance des constantes élastiques de l'alliage à la température considérée, la détermination du caractère des dislocations en jeu. Nous avons pris systématiquement $25^{\circ}$ pour l'angle entre les dislocations parfaites arrivant au nœud et leur vecteur de Burgers, ce qui correspond aux quelques orientations que nous avons déterminées; cet angle est voisin des valeurs médianes des mesures de Loretto sur $\mathrm{Au}-3,3 \% \mathrm{Sn}$ [11], de Gallagher sur $\mathrm{Ag}-12,5 \%$ In [12] et de Ericsson sur Co $-30 \%$ Ni [13].

Nous voudrions enfin souligner que l'on doit procéder à un grand nombre de mesures, et que la considération d'une plage unique, même très nette peut conduire à des erreurs : un échantillon de l'alliage $\mathrm{Ni}-39 \% \mathrm{Cr}$ trempé depuis $1100^{\circ}$ et ayant subi une passe de laminage nous a ainsi donné des nœuds dont le rayon de courbure moyen de $353 \AA$ ( 24 mesures) correspondait à une énergie de $49 \mathrm{erg} / \mathrm{cm}^{2}$ seulement ;

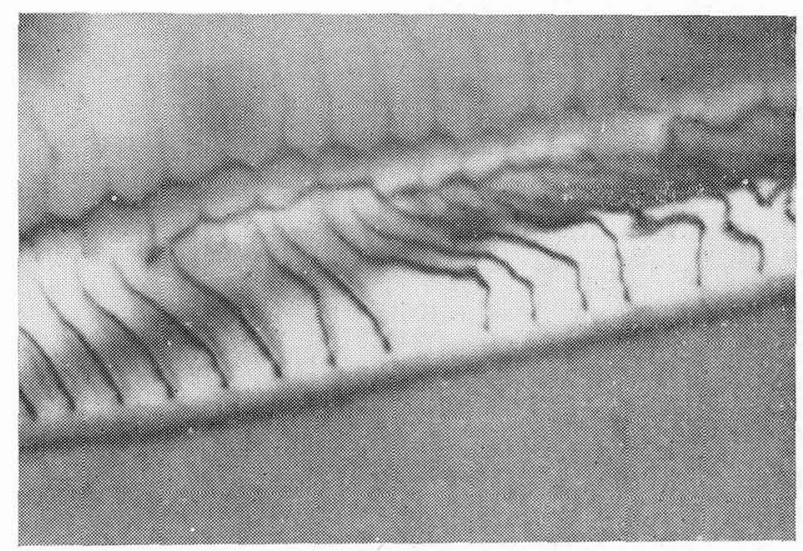

FIg. 4. - Plage unique dans l'alliage nickel$39 \%$ chrome (X 80000$)$. 
ceci s'explique peut-être par un équilibre de température très élevée (Fig. 4). Les nœuds apparents de $244 \AA$ (6 mesures, Fig. 5) que présentaient une plage d'un échantillon $\mathrm{Al}-5 \% \mathrm{Mg}$ après recuit à $500^{\circ}$ et traction de $3 \%$ (probabilité de défaut à $-150^{\circ}=0,004$ )

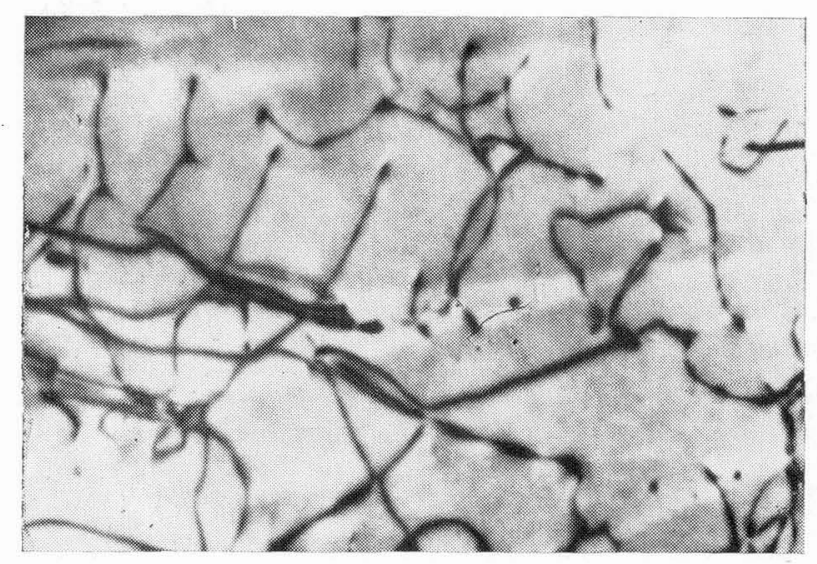

Fig. 5. - Plage unique dans l'alliage aluminium$5 \%$ magnésium (X80 000).

sont sans doute dus à un effet de diffraction électronique (combiné peut-être à une ségrégation d'impuretés, des tensions internes et des restes d'équilibre de température élevée). Ils correspondraient avec les constantes élastiques de cet alliage $(v=0,33$, $G=2,65 \times 10^{11}$ dyne $/ \mathrm{cm}^{2}$ ) à une énergie de $23 \mathrm{erg} / \mathrm{cm}^{2}$ seulement !

L'alliage de nickel à $17,5 \%$ de molybdène et $5,5 \%$ de fer (qui nous avait donné une probabilité de défaut de 0,014 à $-150^{\circ} \mathrm{C}$ ) présente après trempe depuis $1000^{\circ} \mathrm{C}$ des nœuds de $156 \AA$ de rayon de courbure (105 mesures). Avec $v=0,31$ et $G=7,2 \times 10^{11}$ dyne $/ \mathrm{cm}^{2}$

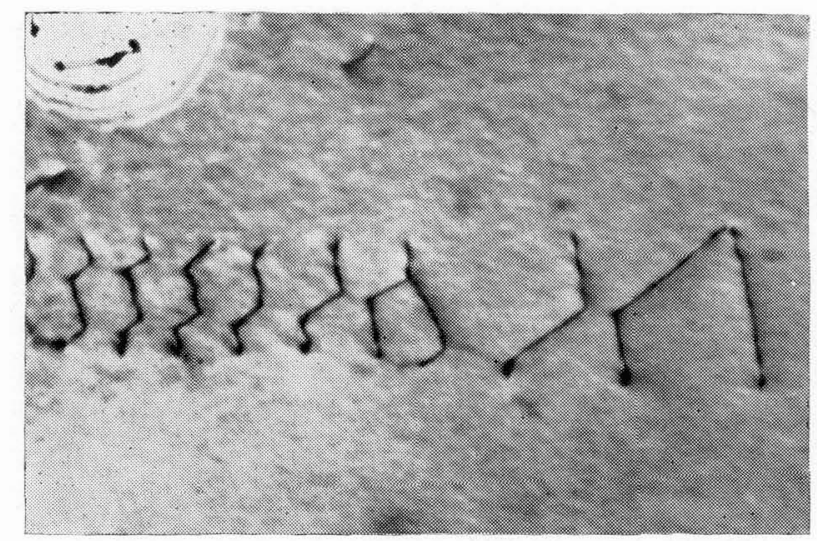

Fig. 6. - Nœuds de dislocations dans l'alliage nickel$17,5 \%$ molybdène- $5,5 \%$ fer (X 80000$)$.
[10] on trouve $\gamma=88 \mathrm{erg} / \mathrm{cm}^{2}$ (Fig. 6). Nous n'avons pas observé de nœuds dissociés sur les alliages nickelcuivre, ni nickel-silicium. Nous poursuivons l'examen d'autres alliages, en essayant d'estimer l'effet de leur pureté, et du mode de traitement créant les nœuds.

Si l'alliage nickel-cobalt où la structure passe du cubique à faces centrées à l'hexagonal compact [13], [14], [15], ou les aciers inoxydables qu'on peut à peine qualifier d'alliages c. f. c. du nickel, ont une énergie de défaut faible [4], les autres alliages où nous avons décelé des défauts assez nombreux par rayons $X\left(\alpha \geqslant 0,012\right.$ à $-150^{\circ}$ par ex.) ont une énergie de défaut de l'ordre de $80 \mathrm{erg} / \mathrm{cm}^{2}$ au moins.

Nous remercions la Direction des Recherches et Moyens d'essais (Ministère des Armées) qui nous accorde son aide matérielle pour le développement de cette étude.

\section{Bibliographie}

[1] Clement (A.) et Coulomb (P.), Physica Status Solidi, 1965, 12, p. 291.

[2] Delehouzée (L.) et Deruyttère (A.), Communication aux journées d'automne de la Société Française de Métallurgie (oct. 1965).

[3] Avec les notations de la référence [1] on a pour les nouvelles mesures radiocristallographiques sur alliages préparés sous vide :

$$
\begin{array}{lll}
\mathrm{Ni}-50 \% \mathrm{Cu} & \alpha_{w}=0,004 & \alpha_{c}=0,003 \\
\mathrm{Ni}-50 \% \mathrm{Pd} & \alpha_{w}=0,005 & \alpha_{c}=0,006 \\
\mathrm{Ni}-15 \% \mathrm{Rh} & \alpha_{w}=0,003 & \alpha_{c}=0,004
\end{array}
$$

[4] Dulieu (D.) et Nutting (J.), Iron and Steel Institute Special Report, 1964, 86, 140.

[5] Irving (C.), Miodownik (A. P.) et Towner (J. M.), J. Inst. Metals, 1965, 93, p. 360.

[6] Brown (L. M.), Philosophical Magazine, 1964, 10, p. 441.

[7] Brown (L. M.) et Thôlen (A. R.), Discussions of the Faraday Society, $1964, \mathbf{n}^{\circ} \mathbf{3 8}$, p. 35.

[8] Siems (R.), Discussions of the Faraday Society, 1964, $\mathrm{n}^{\circ} 38$, p. 42.

[9] Jossang (T.), Stowell (M. J.), Hirth (J. P.) et Lothe (J.), Acta Metal., 1965, 13, p. 279.

[10] American Institute of Physics Handbook, 1957, p. 2-76.

[11] Loretto (M. H.), Phil. Mag., 1964, 10, p. 467.

[12] Gallagher (P. C. J.), J. applied Physics, 1966, 37. 1710.

[13] ERICSSON (T.), Acta Metal., 1966, 14, 853.

[14] MADER (S.), Electron Microscopy and Strength of Crystals, Interscience Publishers, 1963, p. 183.

[15] Köster (E. H.), Thölen (A. R.) et Howie (A.), Phil. Mag., 1964, 10, p. 1093. 